\title{
An SU(4)/SU(2) model as an effort to understand QCD, confinement and asymptotic freedom
}

D. Mastoridis ${ }^{1}-$ K. Kalogirou ${ }^{2}$

\footnotetext{
${ }^{1}$ Ministry of Energy and Environment, Amaliados 17, Athens, Greece, P.O.Box 11523, d.mastoridis@prv.ypeka.gr

${ }^{2}$ Ministry of Energy and Environment, Amaliados 17, Athens, Greece, P.O.Box 11523, k.kalogirou@prv.ypeka.gr
} 


\begin{abstract}
We investigate, whereas the $\mathrm{SU}(4) / \mathrm{SU}(2)$ model, is viable for describing strong interactions. The existing problems of confinement and asymptotic freedom, two phenomena that can not be described by the $\mathrm{SU}(3)$ model, might indicate that we need something "larger" than the SU(3)model. The considered coset SU(4)/SU(2) or the Stiefel manifold $V_{2}\left(C^{4}\right)$ contains an $\mathrm{su}(3)$ algebra, plus additional degrees of freedom that resembes the Feddev-Poppov concept. The richer structure of this coset, give us enough room, to seek for new phenomena, as its dimensionality is 12 . The consideration of the $\mathrm{SU}(4) / \mathrm{SU}(2)$ model flavors firstly the unification of nuclear fields (strong and weak) in an SU(4) model.
\end{abstract}

\title{
1 Acknowledgments
}

We thank F. K. Diakonos ${ }^{3}$ for his extended and helpful critique about our work, which helped and pushed us to develop and examine much more information and aspects, than we had originally thought, for his advices about the structure and style of the presentation of our work and finally for his detailed examination in particle theory and Standard Model.

\section{Introduction}

Undoubtedly, the SU(3)model for describing strong nuclear interactions (QCD) is one of the big discoveries of the 20th century in theoretical physics. Its significance in the understanding of particle's interactions and properties is well known in the area of physics. On the other hand, it can not explain us two significant phenomena

1. Confinement

\section{Asymptotic freedom}

These two phenomena, are essential in our final effort to understand strong interactions or achieve unification. In our oppinion, there is also a third problem, less popular than the other two, that the SU(3) model can not fulfill

3. All the particles described in Standard Model (SM), fermions or bosons, interact with Higg's field, except gluons.

This way, gluons remain massless. Even quarks, that are the fermions associated with strong interactions and are trapped inside hadrons, they interact with Higg's field. In addition, there is a phenomenological belief in physics, that infinite range interactions (as alectromagnetic) give massless bosons, while short range interactions (as weak interactions), give massive bosons. Strong interactions are also short range interactions, which means that we should anticipate massive gluons. But, if someone will try to establish the interaction of gluons with Higg's field, he should consider a triplet Higg's field, where in such case we would have even bigger problems. At this point, we would like to consider two critical questions in order to investigate the above mentioned problems.

$$
\text { Do we need something larger than } S U(3) \text { ? }
$$

Do we need something different structurally, than usual unitary groups as $S U(3)$, that it will still reminds us somehow $S U(3)$ and its properties, but it will also gives us enough room?

\footnotetext{
${ }^{3}$ Department of Physics, University of Athens, Panepistimiopolis, 15771 Athens, Greece
} 
The second question could leads us to a third more general question

Are in general, unitary groups i.e U(1), SU(2), SU(3) enough and capable to describe mathematically, as symmetry groups, the interactions or are they some approximations that should be modified to a new but relevant mathematical scheme?

In order to investigate these questions, it is necessary to be reffered to the FaddeevPopov's [2],[3],[4] and Gribov's concepts [5],[6],[7],[8]. It is evident, that there is a gap between classical Yang-Mills theories and their quantisation as concerned the non abelian case. F-P tried to solve the quantisation problem by adding degrees of freedom, which where adentified as ghost fields. Especially, F-P methods interveve in the fuctional integral, by lifting the usual detrminant to F-P's determinant that automatically drive us to add new fields. But the existence of Gribov's copies means that that F-P methods for quantising non abelian gauge theories is still incomplete. An extensive presentation about F-P and Gribov copies can be found in [1]. Several attempts and modifications gave been considered on these paths, but the problem of Gribov copies, still remains open today and the existence of F-P ghosts tell us that we have failed to formulate a quantum theory with properly fixed physical degrees of freedom [1].

\section{Searching for cantidates, cosets and spheres}

We will try to investigate possible answers to the three above mentioned questions by searching for clues in new mathematical structures that they would be consistent to the standard context. Let us begin with some equivalences such as $U(1) \simeq S^{1}$ and $S U(2) \simeq S^{3}$ where $S^{1}$ and $S^{3}$ are the 1-dimensional and 3 -dimensional spheres respectively. In this spirit the SM can be described as

$$
U(1) \times S U(2) \times S U(3) \simeq S^{1} \times S^{3} \times S^{5} \times S U(2) \simeq S^{1} \times\left(S^{3}\right)^{2} \times S^{5}
$$

We can see that there is a peculiar connection between unitary groups and spheres. Moreover, all the spheres that are presented in Eq. (1) are of odd dimensions. Let us consider, instead of the product of spheres in Eq. (1), a new form as

$$
S^{1} \times S^{3} \times S^{5} \times S^{7}
$$

which is a very attractive form and it comes as the product of odd dimensional spheres with power 1 . Then, this product can be transformed back to unitary groups as

$$
S^{1} \times S^{3} \times S^{5} \times S^{7} \simeq U(1) \times S U(2) \times \frac{S U(4)}{S U(2)}
$$

where in this form, instead of the group $\mathrm{SU}(3)$, we have in its place the coset $\frac{S U(4)}{S U(2)}$. The big question is

Has Eq. (3) the chance to describe SM? 
- Eq. (1): Describes SM but SU(3) fails to give answers about confinment, asymptotic freedom and gluons do not interact with Higg's field. It is very simple and beautiful if the starting point are unitary groups

- Eq. (3):It is very simple and mathematically beautiful if the starting point are spheres and not unitary groups. Moreover, the coset is "larger" than $\mathrm{SU}(3)$ as it contains the necessary su(3) algebra in order to describe our well known gluons (as we shall see further) plus more information. As a result the coset give us room in order to describe new phenomena. In addition, the coset can intaract with a $C^{4}$ double doublet Higg's field. It is clear, in that case, that we expect that the coset could describe strong interactions. This model, firsrly flavors the unification of nuclear fields described by the symmetry group $\mathrm{SU}(4)$ (compact group) and afterwards the unification of nuclear fields with the electromagnetic one, in a model described by U(4) (not compact a problem that will be investigated further)

\section{Cosets and Stiefel maniflods}

It is necessary to try and imagine how a coset looks. We can imagine a coset as a three region structure, a "main building", a "yard" and outside the "yard" where in the "main building" lies the main algebra (in this case the su(3) algebra). Another picture is to imagine the coset as an egg, where we have the yellow part and the white part. Cosets mathematically are related with orbit space (as Gribov copies) and can be also seen from the point of view of Stiefel manifolds as a homogeneous space for the action of a classical group.

Definition: The Stiefel manifold $V_{k}\left(F^{n}\right)$ is the set of all orthonormal frames in $F^{n}$ or the homogeneous space for the action of a classical group in a natural manner. For $F=C^{n}$ it is isomorphical to

$$
V_{k}\left(C^{n}\right)=\frac{S U(n)}{S U(n-k)}
$$

and the dimension is

$$
V_{k}\left(C^{n}\right)=2 n k-k^{2}
$$

In addition cosets are deeply connected with Maurer-Cartan form and equation, where Maurer-Cartan form plays an important role in Cartan's method of moving frames. We must recall the connection of BRST symmetry to the Maurer-Cartan connection. The coset $\frac{S U(4)}{S U(2)}$ breaks as

$$
\frac{S U(4)}{S U(3)} \times \frac{S U(3)}{S U(2)} \simeq S^{7} \times S^{5}
$$

as $S^{7}$ is the isotropy group of $\frac{S U(4)}{S U(3)}$ and $S^{5}$ is the isotropy group of $\frac{S U(3)}{S U(2)}$. In addition all these cosets have simple structure. Specifically, for the cosets we could also write

1. $\mathrm{SU}(4)$ acts transitively on $S^{7}$, with isotropy group $\mathrm{SU}(3)$ 
2. $\mathrm{SU}(4)$ acts transitively on $S^{5}$ via double covering $\mathrm{SU}(4) \rightarrow \mathrm{S} 0(6)$ with isotropy group under the coverimg of the preimage of $\mathrm{SO}(5)$, which can be identified with $\operatorname{Sp}(2)$

3. $\mathrm{SU}(3)$ acts transitively on $S^{5}$, with isotropy group $\mathrm{SU}(2)$

4. $S^{7} \times S^{5}$ is the homogeneous space of $\mathrm{SU}(4)$, with isotropy group $\mathrm{Sp}(2) \cap \mathrm{SU}(3)=\mathrm{SU}(2)$ $\rightarrow \frac{S U(4)}{S U(2)} \rightarrow S^{7} \times S^{5}$

5. $S^{1}$ : Abelian Lie group structure $\mathrm{U}(1)$; the circle group. Topologically equivalent to the real projective line, $R P^{1}$. Parallelizable. $S O(2) \simeq U(1)$.

6. $S^{3}$ :Parallelizable, principal U(1)-bundle over the 2-sphere, Lie group structure $\mathrm{Sp}(1)$, where also $S p(1) \simeq S U(2) \simeq \operatorname{Spin}(3)$

7. $S^{5}$ :Principal U(1)-bundle over $C P^{2}$ equivalent with $S U(3) / S U(2)$ where

$$
C P^{2}=\frac{S U(3)}{S(U(2) \times U(1)}
$$

8. $S^{7}$ : Topological quasigroup structure as the set of unit octonions. Principal $\mathrm{Sp}(1)$ bundle over $S^{4}$. Equivalent to $S U(4) / S U(3)$

9. $\mathrm{U}(4)$ is the only unitary group that can be written as the product of odd dimensional spheres with power 1

The coset $S U(4) / S U(2)$ has a dimension of 12 , which break into the product of $S U(4) / S U(3)$ of dimension 7 and $S U(3) / S U(2)$ of dimension 5 . It is clear that $S^{7}$ can be connected with gluons as it is linked with an octonionic strtucture. But, we can see all the above mentioned about spheres and cosets from the point of view of generators.

\section{Working with generators}

In order to procced further with the generators of the coset $\frac{S U(4)}{S U(2}$, we should start with $\mathrm{SU}(4))$. The generators of $\mathrm{SU}(4)$ are $\lambda_{i}, i=1,2, \ldots 16$. From these 15 matrices, in order to procced with the coset, we must exclude the matrices $\left[\lambda_{1}, \lambda_{2}, \lambda_{3}\right]$ and the coset will have

$$
\frac{S U(4)}{S U(2} \rightarrow\left[\lambda_{4}, \lambda_{5}, \ldots, \lambda_{15}\right]
$$

We break $\lambda_{8}$ and $\lambda_{15}$ as

$$
\begin{aligned}
\lambda_{8} & =\left(\begin{array}{cccc}
0 & 0 & 0 & 0 \\
0 & 1 & 0 & 0 \\
0 & 0 & -1 & 0 \\
0 & 0 & 0 & 0
\end{array}\right)+\left(\begin{array}{cccc}
1 & 0 & 0 & 0 \\
0 & 0 & 0 & 0 \\
0 & 0 & -1 & 0 \\
0 & 0 & 0 & 0
\end{array}\right)=\lambda_{8}^{\prime}+\lambda_{8}^{\prime \prime} \\
\lambda_{15} & =\left(\begin{array}{llll}
0 & 0 & 0 & 0 \\
0 & 1 & 0 & 0 \\
0 & 0 & 1 & 0 \\
0 & 0 & 0 & -2
\end{array}\right)+\left(\begin{array}{cccc}
1 & 0 & 0 & 0 \\
0 & 0 & 0 & 0 \\
0 & 0 & 0 & 0 \\
0 & 0 & 0 & -1
\end{array}\right)=\lambda_{15}^{\prime}+\lambda_{15}^{\prime \prime}
\end{aligned}
$$


Then $\left[\lambda_{6}, \lambda_{7}, \lambda_{8}^{\prime}\right]$ consist an $\mathrm{su}(2)$ algebra as

$$
\left[\lambda_{6}, \lambda_{7}, \lambda_{8}^{\prime}\right] \rightarrow\left(\begin{array}{ll}
0 & \\
& s u(2)
\end{array}\right)
$$

and $\left[\lambda_{6}, \lambda_{7}, \lambda_{8}^{\prime}, \lambda_{11}, \lambda_{12}, \lambda_{13}, \lambda_{14}, \lambda_{15}^{\prime}\right]$ consist an su(3) algebra as

$$
\left[\lambda_{6}, \lambda_{7}, \lambda_{8}^{\prime}, \lambda_{11}, \lambda_{12}, \lambda_{13}, \lambda_{14}, \lambda_{15}^{\prime}\right] \rightarrow\left(\begin{array}{ll}
0 & \\
& s u(3)
\end{array}\right)
$$

As concerned the existence of $\lambda_{8}^{\prime}, \lambda_{8}^{\prime \prime}, \lambda_{15}^{\prime}, \lambda_{15}^{\prime \prime}$, either we can keep as above mentioned, where $\lambda_{8}^{\prime \prime}, \lambda_{15}^{\prime \prime}$ ) will be additional auxiliary fields or by the beginning we can change basis for $\left[\lambda_{3}, \lambda_{8}, \lambda_{15}\right] \rightarrow\left[\lambda_{3}^{\prime}, \lambda_{8}^{\prime}, \lambda_{15}^{\prime}\right]$, where the new basis will be expressed as linear combination of the components of the old basis. Furthermore, using appropriate coefficients, we can double the $\mathrm{su}(2)$ algebra and form an $\mathrm{su}(2) \times \mathrm{su}(3)$ algebra. As concerned, the rest generators of the coset that do not participate in the $\mathrm{su}(3)$ algebra i.e $\left[\lambda_{4}, \lambda_{5}, \lambda_{9}, \lambda_{10}\right]$, we have 4 auxiliary fields. In the case that we include $\lambda_{8}^{\prime \prime}, \lambda_{15}^{\prime \prime}$ that must be interoretated In F-P concept there are additional degrees of freedom that do not count as natural . But, in our case these 4 additional "fields" seem quite natural. As we do not have the chance to "look into" hadrons, there is room to consider that there exist 8 gluons plus new additional bosons that we will call them as residual gluons. An interesting point of this analysis, is that we can have a fresh look, as it comes from the spheres. The full coset has 12 dimensions as

$$
\frac{S U(4)}{S U(2)} \simeq S^{7} \times S^{5} \longrightarrow 12 \rightarrow 7+5
$$

If we analyse these dimensions, the 12 generators will break as follows

- Some of the generators of the $\mathrm{su}(3)$ algebra that represent gluons, would be assigned to $S^{7}$ and some others to $S^{5}$

- Some of the remaining generators that do not produce the su(3) algebra and represent the auxiliary fields would be assigned to $S^{7}$ and some others to $S^{5}$

As a consequence we face two possible interpretations

a) If the algebra su(3) represents gluons, then there are two types of gluons, the ones that comes from $S^{7}$ and the others to $S^{5}$, with differences among them.

b) The gluons are same among them, but "come" as a linear combination of two different field arising from $S^{7}$ and $S^{5}$

Which case is valid, will tell us how to treat the coupling constants.

The interesting point, apart from the existence of the $\mathrm{su}(3)$ algebra that it is used to represent gluons, is the existence of the extra generators $\lambda_{4}, \lambda_{5}, \lambda_{9}, \lambda_{10}$ plus the auxiliary generators $\lambda_{8}^{\prime \prime}, \lambda_{15}^{\prime \prime}$. We will assign fileds to these generators as

$$
\lambda_{4} \rightarrow \varphi_{1} \quad \lambda_{5} \rightarrow \varphi_{2} \quad \lambda_{9} \rightarrow \chi_{1} \quad \lambda_{10} \rightarrow \chi_{2} \quad \lambda_{8}^{\prime \prime} \rightarrow \omega_{1} \quad \lambda_{15}^{\prime \prime} \rightarrow \omega_{2}
$$


Those $4+2$ generators, all together do not form any particular algebra, but they have some interesting properties as

1. $\varphi_{1}=i \varphi_{2} \quad \chi_{1}=i \chi_{2}$

2. the pairs $\varphi_{1}, \varphi_{2}$ and $\chi_{1}, \chi_{2}$ are Grassmann numbers (variables), while the pair $\omega_{1}, \omega_{2}$ are not

3. the pairs $\varphi_{1}, \omega_{1}$ and $\varphi_{2}, \omega_{1}$ and $\chi_{1}, \omega_{2}$ and $\chi_{2}, \omega_{2}$ are also Grassmann numbers

4. the triplets $\varphi_{1}, \varphi_{2}, \omega_{1}$ and $\chi_{1}, \chi_{2}, \omega_{2}$ are also Grassmann numbers

5. there exist $S_{1}, S_{2}$ matrices that transmutes $\varphi_{1}, \varphi_{2}$ and $\chi_{1}, \chi_{2}$ to $\omega_{1}, \omega_{2}$ respectively. This property explains why $\omega_{1}, \omega_{2}$ will be assigned to auxiliary fields as

$$
\begin{gathered}
S_{1} \varphi_{1}=\omega_{1} \quad S_{2} \chi_{1}=\omega_{2} \\
i S_{1} \varphi_{2}=\omega_{1} \quad i S_{2} \chi_{2}=\omega_{2}
\end{gathered}
$$

with

$$
S_{1}=\left[\begin{array}{cccc}
0 & 0 & -1 & 0 \\
0 & 0 & 0 & 0 \\
1 & 0 & 0 & 0 \\
0 & 0 & 0 & 0
\end{array}\right] \quad S_{2}=\left[\begin{array}{cccc}
0 & 0 & 0 & -1 \\
0 & 0 & 0 & 0 \\
0 & 0 & 0 & 0 \\
1 & 0 & 0 & 0
\end{array}\right]
$$

Those properties, form a BRST type symmetry. The extra fields $\varphi_{1}, \varphi_{2}, \chi_{1}, \chi_{2}$ reminds us the F-P concept, except the fact that these fields are not come up from the quantisation problem and fixing of the determinant, but rather they come naturally from the structure of the coset's symmetry and vice-versa. Moreover, as we will see, they are no longer ghost fields. In addition, the coset direct us to a Cartan-Maurer type connection, which direct us to a BRST type symmetry. Even, Gribov's copies, which are deeply connected with gauge orbits, they can be better understood and handled, under the shelter of the concept of the coset.

\section{Discussion}

\subsection{Bosons}

The problem that must be solved, is the interpretation of the extra fields. As far as at this part, we have two type of fields, our ordinary $G_{\mu}$ fields (associated with the su(3) algebra inside the coset) that we will still interpretate as gluons, the extra fields $\varphi_{1}, \varphi_{2}, \chi_{1}, \chi_{2}$ and the $\omega_{1}, \omega_{2}$ auxiliary ones. Let us interpretate the extra fields $\varphi_{1}, \varphi_{2}, \chi_{1}, \chi_{2}$ as new bosons that we will call them as residual gluons. In our oppinion, these residual gluons, are deeply connected with the residual nuclear field and the mechanism under pions are created. Our current picture in the usual context of physics, is that pions (among with rho and omega mesons) mediate as carriers between hadrons. This picture might indicate the existence of extra bosons (apart from gluons) In addition, these residual gluons could play a fundamental role in the forming and disquisition of mesons and hadrons. Especially, as the residual strong fields occurs by the exchange of spinless pions, those virtual mesons possess a fundamental role along all the other known mesons. We could imagine a picture, where our usual gluons "live" in the "central building" and the residual gluons in the "yard" as the coset dictates. Our usual gluons are responsible for the strong interactions, while the residual ones are responsible for the residual nuclear field. But, at the same time gluons and residual gluons are interacting. Those two fields are imprisoned in the structure defined by the coset and this is the reason why the combined field behaves differently (hadronic prison) than the weak nuclear and the electromagnetic fields. Followingly, we should investigate, how the above mentioned picture, will affect the unification. As, 


$$
S^{1} \times S^{3} \times S^{5} \times S^{7} \simeq U(4) \simeq \simeq S U(4) \times U(1)
$$

we can see that the model flavors firstly the unification of strong with weak nuclear field in a unified nuclear field described by $12+3=15$ bosons and afterwards this unified nuclear field will be unified with the electromagnetic one. Moreover, logic might indicate that we will have one massless boson (photon) and 15 massive bosons, which follows the phenomenological rule that short range fields correspond to massive bosons. Nevertheless, this question (about which bosons are massive and which are not) could be answered only by solving for the eigenvalues. As $\mathrm{SU}(4)$ is a compact group, we should be able to find the coupling constants of the nuclear fields. At the same time. as $U(4)$ is not a compact group, the coupling constant for the electromagnetic field could be found by a flip-flop mechanism or as a free parameter of the model. In the current context of physics, we are used to symbolise the coupling constant of electromagnetic fields as $g_{1}$ (U(1)), of weak nuclear as $g_{2}(\mathrm{SU}(2))$ and of strong nuclear as $g_{3}(\mathrm{SU}(3))$. In order to avoid any misunderstandings, we will keep the current coupling constants symbols for electromagnetic and weak nuclear field, but we will use different symbols for the coupling constants assigned to $\mathrm{SU}(3), \mathrm{SU}(4)$ as

$$
k_{3} \mapsto S U(3) \quad k_{4} \mapsto S U(4)
$$

In this spirit, the coset will have a combined constant as

$$
\begin{gathered}
\frac{S U(4)}{S U(2)} \simeq \frac{S U(4)}{S U(3)} \times \frac{S U(3)}{S U(2)} \\
\frac{k_{4}}{g_{2}}\left|\mapsto \frac{k_{4}}{k_{3}}\right|+\frac{k_{3}}{g_{2}} \mid
\end{gathered}
$$

The question is if our well known coupling constant $g_{3}$ corresponds to $\frac{k_{4}}{g_{2}}$ or if $g_{3}$ comes after a part of the mixture of the coupling constants $\frac{k_{4}}{k_{3}}$ and $\frac{k_{3}}{g_{2}}$. In the second case, the fields that correspond to the $\mathrm{su}(3)$ algebra produced by the mixture of the fileds will be assigned the $g_{3}$ coupling constant. In each case, if our consideration is valid and assume that all the coupling constants involved are decreasing, the quotient under certain constraints, could increase, expaining this way the asyptotic freedom phenomenon. In this spirit, a new covariant derivative should be formed to be connected to the coset $\frac{S U(4)}{S U(2)}$ as

$$
D_{\mu}=\partial_{\mu}-i G_{\mu}-i[\quad, \quad]
$$

where in the Lie bracket, we should find the auxiliary fields in order to close the $\mathrm{su}(4)$ algebra, in the form $\left[R_{i}, R_{j}\right]$ or $\left[\partial_{i}, S_{j}\right]$ where $R_{i}$ are the extra fields assigned as residual gluon fields and auxiliary fields. The Lie bracket, automatically suggests that the 
propagator connected to the covariant derivative, will be affected by the extra fields (we can found a similarity with Gribov and Faddeev-Popov ghost theories). All the above mentioned analysis, leads to a prediction of our approach, that we should consider a $\varphi \in$ $C^{4}$ model, where this field is not anymore an ad-hoc consideration but ruther is indicated by the geometry, where $\varphi$ will be written as

$$
\varphi=\left(\begin{array}{l}
\varphi_{1}+i \varphi_{2} \\
\varphi_{3}+i \varphi_{4} \\
\varphi_{5}+i \varphi_{6} \\
\varphi_{7}+i \varphi_{8}
\end{array}\right)
$$

This $C^{4}$ model, allows the strong nuclear field to fully participate in the Higg's mechanism. In addition, the quatraplet Higg's field $\varphi$, can be seen as "doublet doublet". In this sense, if in the well known $C^{2}$ G. W. S model we consider the field $\varphi$ as

$$
\varphi=\left(\begin{array}{l}
\varphi^{+} \\
\varphi^{o}
\end{array}\right)
$$

where we denote this way the "charged" and "neutral " part, in the $C^{4}$ model, we should incude new symbols to denote whereas the components of the $C^{4}$ field interacts "charged" or "netral" in the sense of the strong nuclear "charge". In the case of the full covariant derivative, including nuclear and electromagnetic fields, there will be a full hypercharge $Q^{\prime}$ with $Q^{\prime} \varphi=0$ derived from the combination $\left[I, T_{3}, T_{8}, T_{15}\right]$, which will break as

$$
Q^{\prime} \varphi \rightarrow Q \varphi+Q^{\prime \prime} \varphi
$$

where $\mathrm{Q}$ is the usual hypercharge and $Q^{\prime \prime}$ the hypercahrge corresponded to the coset.

\subsection{Fermions}

The choice of a quatraplet field, automatically means for the case of fermions, that we have to consider a unified fermion quatraplet as

$$
f=\left(\begin{array}{l}
l_{i} \\
\nu_{i} \\
u_{i} \\
d_{i}
\end{array}\right)_{x} \rightarrow \frac{\left(\begin{array}{l}
l_{i} \\
\nu_{i}
\end{array}\right)_{L}}{\left(\begin{array}{l}
u_{i} \\
d_{i}
\end{array}\right)_{B}}
$$

where $i=1,2,3$ as $l_{1,2,3}=(e, \mu, \tau), n_{1,2,3}=\left(\nu_{e}, \nu_{\mu}, \nu_{\tau}\right), u_{i}=$ (up,charm,top), $d_{i}=$ down, strange, bottom) and $\mathrm{x}$ is a new partivle number that unifies $\mathrm{L}$ and $\mathrm{B}$. Moreover, in this spirit, we will be able to reduce the existing particle numbners $\left(Q, T_{3}, I_{3}, S, C, B^{\prime}, T, L, B\right)$ to just six, which seems logical, due to the fact that there are six "charges" in SM, one for elecrtomagnetism, 2 "charges" for weak nuclear and three "charges" for strong nuclear 
field. Six "charges" means six particle numbers. These three particle numbers can be seen as

$$
\begin{aligned}
u p & \rightarrow A \\
\text { charm } & \rightarrow B \longrightarrow T_{3} \uparrow \\
\text { top } & \rightarrow C \\
\text { down } & \rightarrow A \\
\text { strange } & \rightarrow B \longrightarrow T_{3} \downarrow \\
\text { bottom } & \rightarrow C
\end{aligned}
$$

$$
\begin{aligned}
e & \rightarrow A \\
\mu & \rightarrow B \longrightarrow T_{3} \uparrow \\
\tau & \rightarrow C \\
\nu_{e} & \rightarrow A \\
\nu_{\mu} & \rightarrow B \longrightarrow T_{3} \downarrow \\
\nu_{\tau} & \rightarrow C
\end{aligned}
$$

where A, B, C are new particle numbers, and the quarks and leptons are distinguished by L, B. But as x unifies L, B, we just need six particle numbers as

$$
\left(Q, A, B, C, T_{3}, x\right)
$$

\section{Concusion}

We have investigated QCD, confinment and asyptotic freedom,with a new prespective, in order to find something richer than the $\mathrm{SU}(3)$, which is the symmtry group that we currently use to describe strong interactions. We have considered the coset $\mathrm{SU}(4) / \mathrm{SU}(2)$ which can give us very important properties, that in oup oppinion, could serve us to make a big step in understanding strong interactions and the missing properties of confinment and asyptotic freedom. But, this manuscript is just the beginning of such an enterprise, as a lot of steps must be fulfilled in order to have a positive answer as

1. An exact covariant derivative compatible with the concept of coset is needed

2. Using this covariant derivative, we should proceed in detail with the unification scheme of nuclear fields, the calculation of their coupling constants and the calculation of the fields eigenvalues

3. We should present the fields propagations of gluons and residual gluons 


\section{References}

\section{References}

[1] N. Vandersickel, Daniel Zwanziger, The Gribov problem and QCD dynamics, arXiv:1202.1491v2 .

[2] Faddeev, L D and Popov,, Feynman Diagrams for the Yang-Mills Field, Phys. Lett. B25: 29-30, V N (1967).

[3] Faddeev, L D (1969), Feynman integral for singular Lagrangians. , Theor. Math. Phys. 1: 1. doi:10.1007/bf01028566..

[4] Popov, V N and Faddeev,, English translation: Perturbation theory for gaugeinvariant fields, Kiev Inst. Theor. Phys. Acad. Sci. preprint ITP 67-36, Reprinted e.g. in 50 Years of Yang-Mills Theory, ed. G 't Hooft, World Scientific 2005.

[5] V.N. Gribov, Gauge Theories and Quark Confinement, collection of works, Phasis Publishing house, Moscow, 2001 [www.aha.ru/〜 phasis].

[6] V.N. Gribov, K. Yc, Space-time description of the hadron interaction at high energies,in: Proceedings of the 1st ITEP School, vol. 1 Elementary particles, Moscow, 1973, p. 65 (in Russian); hep-ph/0006158.

[7] V.N. Gribov, Possible solution of the problem of quark confinement,preprint LU-TP91-7, Presented at Perturbative QCD Workshop, Lund, Sweden, May 21-24, 1991.

[8] V.N. Gribov and J. Nyiri, Quantum electrodynamics,(Gribov lectures on Theoretical Physics), Cambridge University Press, 2001. 